\title{
Evaluación de la efectividad del Triclabendazol como fasciolicida en caprinos de la provincia de Neuquén, Argentina
}

\author{
Centelles $\mathrm{I}^{1}$, Martínez $\mathrm{A}^{2}$, Cabrera $\mathrm{R}^{2}$, Castillo $\mathrm{B}^{3}$, Larroza $\mathrm{M}^{2 *}$
}

1. Agencia de Extensión Rural, Instituto Nacional de Tecnología Agropecuaria (INTA), Chos Malal, Provincia de Neuquén, Argentina.

2. Grupo de Salud Animal, INTA Bariloche, Provincia de Río Negro, Argentina.

3. Agencia de Producción y Sanidad, Secretaria de Desarrollo territorial y ambiente, Colipilli Provincia de Neuquén, Argentina.

* Correspondencia: Marcela Larroza, Estación Experimental Agropecuaria Bariloche, INTA, Modesta Victoria 4450, 8400 San Carlos de Bariloche, Argentina. E-mail: larroza.marcela@inta.gob.ar

Recibido: 19 Mayo 2021. Aceptado: 6 Septiembre 2021. Disponible en línea: 2 Noviembre 2021

Editor: P. Beldomenico

RESUMEN. Debido al uso frecuente del triclabendazol (TCBZ) por parte de los productores, sumado al reporte reciente de resistencia de Fasciola hepatica al fármaco detectada en rodeos y majadas de Neuquén, se llevó a cabo el presente trabajo con el objetivo de evaluar la eficacia del TCBZ como fasciolicida en caprinos bajo un sistema de producción trashumante del norte de la provincia de Neuquén, Argentina. La eficacia se evaluó mediante un test de reducción de conteo de huevos de $F$. hepatica en caprinos Criollo Neuquino parasitados naturalmente. Se utilizaron dos grupos de animales: un Grupo Control ( $n: 10)$ que no recibió tratamiento y un Grupo Tratado ( $\mathrm{n}: 10$ ) al que se le administró TCBZ al 10\% (Biofasiolex T10-Biogénesis Bagó ${ }^{\circledR}$ ) vía oral a dosis de $10 \mathrm{mg} / \mathrm{kg}$ PV. Al día 0 y 21 post tratamiento, se tomaron muestras de materia fecal para análisis coproparasitológicos. Se evidenció una reducción del conteo de huevos de $F$. hepatica del $100 \%$, indicando el mantenimiento de la eficacia del fasciolicida en los caprinos evaluados. Se discuten los alcances del uso del test de reducción de conteo de huevos en zonas con producción trashumante del ganado.

SUMMARY. Evaluation of the efficacy of Triclabendazol to treat liver worm in goats in Nequen province, Argentina. Because of the frequent use of triclabendazole TCBZ by farmers and the recent reports of Fasciola hepatica resistance to this drug in herds and flocks from Neuquén, we carried out this study with the aim to evaluate the efficacy of TCBZ as fasciolicide in goats under a transhumance system from northern of Neuquén, Argentina. A fecal egg count reduction test (FECRT) was performed on Creale Neuquino goats naturally parasitized with $F$. hepatica. Twenty goats were divided into two groups: A control group ( $\mathrm{n}: 10)$ which was left untreated, and a treatment group ( $\mathrm{n}: 10$ ) that was dosed orally with TCBZ 10\% (Biofasiolex T10-Biogénesis Bagó ${ }^{\circledR}$ ). On Day 0 and Day 21 posttreatment, fecal samples were taken and analyzed by coproscopical examination. According to the FECRT, drug efficacy was $100 \%$ and confirms that TCBZ maintains its efficacy against $F$. hepatica on goats. The usefulness of the FECRT in these zones under transhumance livestock production is discussed.

Palabras clave: Caprinos; Trashumancia; Eficacia; Fasciola hepatica; Test de reducción de conteo de huevos

Keywords: Goats; Transhumance; Efficacy; Fasciola hepatica; Egg count reduction test

\section{Introducción}

La fasciolosis, producida por el parásito trematodo Fasciola hepatica (Fh), es una enfermedad parasitaria que afecta herbívoros y omnívoros, y ocasionalmente al hombre (Acha y Szyfres, 1985). Su distribución varía notablemente de acuerdo a las regiones geográficas, parasitando en general a animales de regiones con lluvias moderadas a intensas, aunque también se observa en regiones más secas, a lo largo de arroyos, mallines o humedales, y por canales de riego que albergan a caracoles del género Lymnaea, que actúan como huéspedes intemediarios en el ciclo biológico de Fh (Olaechea, 2007).

En la Patagonia Argentina, el parásito se encuentra endémicamente en establecimientos ganaderos en las zonas de cordillera y precordillera. En estos establecimientos, es frecuente el uso de triclabendazol (TCBZ) como fasciolicida debido a su reconocida eficacia contra estadios maduros e inmaduros de Fh, baja toxicidad, amplio espectro y poder residual, realizando sistemáticamente una o dos desparasitaciones anuales con TCBZ, sin rotación de principios activos ni manejo de pastoreo (Aguilar y Alvarez, 2017). 
Mediante un estudio epidemiológico realizado a productores de los parajes de Manzano Amargo y Guañacos del norte de la provincia de Neuquén, se determinó que el $78 \%$ de los productores utilizaban rutinariamente el TCBZ para el control de la fasciolosis en caprinos; sin embargo, los resultados obtenidos mediante estudios coproparasitológicos indicaron que el $40,8 \%$ de los animales continuaban parasitados a pesar del plan de desparasitación utilizado por los productores (Alberghini, 2019). Más aún, frecuentemente los tratamientos antiparasitarios en la región se realizan sin supervisión veterinaria, sin diagnóstico coproparasitológico previo, ni rotación de principios activos (Alberghini, 2019).

Como consecuencia de este uso indiscriminado, en el año 2011 se reportó la resistencia antiparasitaria de $\mathrm{Fh}$ al TCBZ en un establecimiento ganadero bovino de la provincia de Neuquén (Olaechea et al., 2011). Posteriormente se han corroborado otros casos de resistencia de Fh al TCBZ en bovinos y ovinos en diversos establecimientos de Patagonia (Larroza, datos no publicados). Sin embargo, hasta la fecha no se han realizado estudios de eficacia de antiparasitarios fasciolicidas en caprinos; debido a estos antecedentes, se llevó a cabo una prueba de eficacia a campo del TCBZ en caprinos del Norte Neuquino parasitados naturalmente.

\section{Materiales y Métodos}

El estudio se realizó en un hato caprino de raza Criollo Neuquino, perteneciente a un criancero de la comunidad mapuche Huayquillan, ubicada en el paraje Colipilli $\left(37.7^{\circ} \mathrm{S}, 70.4^{\circ} \mathrm{W}\right)$, Provincia de Neuquén, Argentina (Figura 1). Según datos obtenidos en un estudio epidemiológico, el productor manifestó que a pesar de los tratamientos con TCBZ realizados en el año, se observaba presencia de parásitos adultos en el hígado de los caprinos faenados. El hato era manejado bajo un sistema de producción tradicional extensivo con trashumancia, lo cual implica el movimiento de los animales desde los campos bajos de invernada a los valles cordilleranos de veranada en busca de mejores pasturas.

Para realizar el estudio, se utilizaron caprinos Criollo Neuquino de 2 a 6 dientes, identificados con caravanas, que no hubiesen sido desparasitados al menos durante los tres meses previos al ensayo. Tres semanas antes del inicio del trabajo, se realizó un muestreo y análisis de materia fecal con la finalidad de confeccionar los grupos, de acuerdo al conteo de huevos de Fh.

Con los animales seleccionados se conformaron dos grupos experimentales correspondientes a un grupo control (GC) compuesto por 10 animales a los cuales no se les realizó ningún tratamiento, y un grupo tratado (GT) de 10 animales a los que se les administró TCBZ al 10\% (Biofasiolex T10 - Biogénesis Bagó) por vía oral en dosis de $10 \mathrm{mg} / \mathrm{kg}$ PV. Al inicio del estudio (Día 0), todos los animales fueron pesados mediante una balanza electrónica y se administró TCBZ al GT de acuerdo a su peso corporal. Las muestras de materia fecal se tomaron de manera individual directamente del recto los días 0 y 21 post tratamiento, y se mantuvieron refrigeradas hasta su envío y procesamiento en el laboratorio de Parasitología de INTA Bariloche. Para la identificación y conteo de huevos de Fh por gramo de materia fecal (HpgFh), se utilizó la técnica de Sedimentación - Filtración (Fiel et al., 2011).

El cálculo de la eficacia se realizó a través de la comparación de los conteos de HpgFh en los caprinos antes y después del tratamiento, según el test de reducción del conteo de huevos (TRCH), de acuerdo al protocolo establecido por la Asociación Mundial para el Desarrollo de la Parasitología Veterinaria (WAAVP) (Coles et al., 1992).

Para el cálculo del porcentaje de reducción del conteo de HpgFh se utilizó el promedio de cada grupo de muestras colectadas a los 21 días post-tratamiento, de acuerdo a la siguiente fórmula:

\section{P. R. C. H. $(\%)=[(C-T) / C] \times 100$}

Donde $C$ es la media aritmética del grupo control sin tratamiento, y $\mathrm{T}$ es la media aritmética del grupo tratado 21 días post-tratamiento (Coles et al., 1992).

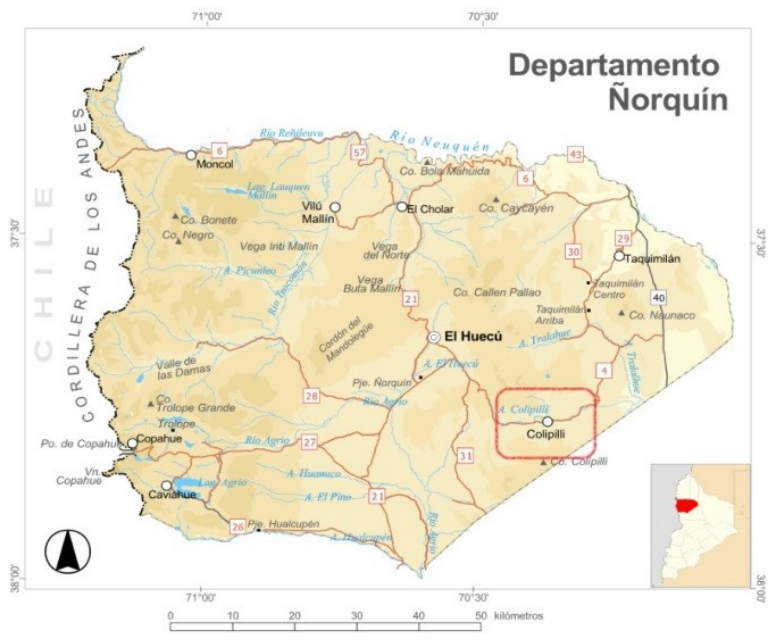

Figura 1. Ubicación del predio en estudio en la Provincia de Neuquén, Argentina

\section{Resultados}

Los resultados individuales de HpgFh obtenidos de cada animal se presentan en la Tabla 1 . En el día 0 , el promedio HpgFh del GC fue de 28.9 (rango: 11-60), y en el GT de 30.7 (rango: 14-67). En el día 21 posttratamiento, el promedio del GC fue de 16.3 (rango: 521), mientras que no se observaron huevos de $\mathrm{Fh}$ en las muestras del GT para esta fecha. De acuerdo a estos resultados, el porcentaje de reducción del conteo de 
HpgFh en los caprinos evaluados luego del tratamiento con TCBZ fue del $100 \%$.

Tabla 1. Huevos de Fasciola hepatica por gramo de materia fecal (HpgFh) al día del tratamiento (0) y 21 días posttratamiento (21) con triclabendazol en un test de reducción del conteo de huevos en caprinos del Norte de Neuquén, Argentina.

\begin{tabular}{|c|c|c|c|}
\hline & Animal & Día 0 & Día 21 \\
\hline \multirow{10}{*}{ Grupo Control } & 1 & 11 & 5 \\
\hline & 2 & 12 & 59 \\
\hline & 3 & 14 & 15 \\
\hline & 4 & 16 & 18 \\
\hline & 5 & 21 & 12 \\
\hline & 6 & 32 & 4 \\
\hline & 7 & 33 & 25 \\
\hline & 8 & 38 & 6 \\
\hline & 9 & 52 & 5 \\
\hline & 10 & 60 & 14 \\
\hline \multirow{10}{*}{ Grupo tratado } & 11 & 19 & 0 \\
\hline & 12 & 58 & 0 \\
\hline & 13 & 28 & 0 \\
\hline & 14 & 67 & 0 \\
\hline & 15 & 20 & 0 \\
\hline & 16 & 14 & 0 \\
\hline & 17 & 14 & 0 \\
\hline & 18 & 35 & 0 \\
\hline & 19 & 21 & 0 \\
\hline & 20 & 31 & 0 \\
\hline
\end{tabular}

\section{Discusión}

En el presente estudio se confirmó que el TCBZ a dosis de $10 \mathrm{mg} / \mathrm{kg}$ PV tuvo una eficacia del $100 \%$ en la reducción del número de huevos de Fh en los caprinos tratados del Norte de la Provincia de Neuquén. Con estos resultados inferimos que las fallas en los tratamientos mencionados por los productores de la zona podrían deberse a errores de manejo, tales como dosificación según estimación visual del peso, instrumental descalibrado o en malas condiciones, uso de productos no adecuados, entre otros, y no a una reducción per se en la efectividad antiparasitaria del TCBZ.

En Argentina, la resistencia de Fh al TCBZ fue reportada por primera vez en bovinos de la provincia de Neuquén (Olaechea et al., 2011), seguida por registros de casos en la provincia de Chubut en bovinos (Prieto, 2016), mientras que los hallazgos de resistencia y fallas de la eficacia del TCBZ a campo han aumentado progresivamente en los últimos años en ovinos y bovinos de Patagonia Norte (Larroza, datos no publicados). A nivel mundial, desde el primer reporte de resistencia del TCBZ a Fh en ganado en Australia (Overend y Bowen, 1995), se han reportado casos en Escocia (Mitchell et al., 1998), Gales (Thomas et al., 2000), Países Bajos (Moll et al., 2000), España (Alvarez-Sánchez et al., 2006), Irlanda (Mooney et al. 2009), Nueva Zelanda (Hassell y Chapman, 2012) y Perú (Ortiz et al., 2013; Cabada et al. 2016), evidenciando que la resistencia de Fh al TCBZ es un problema emergente en todo el mundo. Específicamente en caprinos, son escasos los antecedentes a nivel internacional encontrándose sólo un estudio en el sur de Brasil que reportó resistencia de Fh al TCBZ, encontrando un 57,3\% de eficacia fasciolicida (Oliveira et al., 2008).

En la zona Norte de Neuquén es característico el sistema de producción de tipo trashumante, el cual consiste en una práctica de desplazamiento de animales desde los campos bajos de "invernada" a los valles altos de las "veranadas" cordilleranas donde es frecuente el pastoreo conjunto entre varios hatos y rodeos (Lanari et al., 2005). Este movimiento de animales, tiene como consecuencia el riesgo epidemiológico que implica el desplazamiento entre los distintos lugares de pastoreo, pudiendo propagar enfermedades contagiosas a lo largo de su ruta de arreo y/o lugar de pastoreo (Abiola et al., 2005). Sin embargo, a pesar de que los caprinos bajo estudio pastorean en un valle de veranada a menos de $30 \mathrm{~km}$ del establecimiento bovino que tuvo antecedentes de resistencia de Fh al TCBZ (Olaechea et al., 2011), los resultados presentados aquí no indicarían una diseminación de la cepa resistente entre ambas especies de ganado. Esta situación puede deberse a que los valles ubicados en los campos de veranada utilizados por bovinos y ovinos, poseen un sistema natural de irrigación diferente entre ambos y sin posibilidad de conexión debido a la distancia, disminuyendo así la probabilidad de dispersión y comunicación por parte de los huéspedes intermediarios portadores de dicha cepa.

Según un estudio reciente, el TCBZ es utilizado como fasciolicida en caprinos dos o tres veces por año por el $78 \%$ de los productores de la zona en Neuquén (Alberghini, 2019). Considerando los antecedentes mencionados, y su uso continuo como fasciolicida, se evidencia la necesidad de evaluar periódicamente la acción de este fármaco contra Fh, con el fin de detectar en forma temprana el desarrollo de poblaciones de parásitos resistentes a TCBZ. Ante la sospecha de baja eficacia antiparasitaria, existen diferentes métodos 
para la detección de resistencia, entre ellos el TRCH es una valiosa herramienta para realizar a campo debido a su practicidad y bajo costo (Fiel et al., 2011). Su implementación frecuente permitiría corroborar la eficacia de los productos en uso, así como también la actividad de principios activos poco utilizados por los crianceros hasta el momento. Asimismo, la realización de estudios de eficacia fasciolicida en distintos predios, otorgaría evidencia sobre posibles fallas de eficacia o resistencia antiparasitaria a nivel regional.

En conclusión, se verificó que la eficacia del TCBZ como fasciolicida es del $100 \%$ en los caprinos Criollo estudiados del norte de la provincia de Neuquén. Para mantener esta efectividad y retrasar los procesos de resistencia antiparasitaria, se deben evitar las dosificaciones rutinarias, administrando los tratamientos según los resultados de los análisis coproparasitológicos periódicos, y realizar rotaciones con antiparasitarios con diferentes principios activos (Nari, 2003; Lanusse, 2009). Se recomienda también adecuar el manejo sanitario al sistema de producción, y sostener los programas de asesoramiento técnico, capacitando sobre el uso correcto de los antiparasitarios y el manejo del pastoreo. La utilización de métodos integrados de control, basados en las características epidemiológicas regionales permitirán extender la vida útil de los fármacos disponibles para el tratamiento de la fasciolosis.

\section{Agradecimientos.}

Agradecemos a la comunidad Mapuche Huayquillan por permitir la realización de este estudio, particularmente al productor Arnaldo y Antonio Huayquillan y sus familias, por la colaboración brindada en el trabajo a campo. También agradecemos a la AER INTA Chos Malal por la colaboración en los muestreos a campo, y a Biogénesis por suministrarnos el Biofasiolex T10 utilizado. Este trabajo fue financiado por proyectos INTA: PD 1104 "Estudios sobre la generación de resistencia a los antimicrobianos"; PE 1002 "Producción y sanidad de los Pequeños Rumiantes" y FONTAGRO "Produccion de la Agricultura Familiar".

\section{Referencias}

Abiola FA, Teko-Agbo A, Biaou C, Niang M. 2005. Socioeconomic and animal health impact of transhumance. Ecole Inter-Etats des Sciences et Médecine Vétérinaires. Conférence OIE. Pp. 85-109.

Acha PN, Szyfres B. 1985. Zoonosis y enfermedades transmisibles comunes al hombre y a los animales. Organización Panamericana de la Salud y Organización Mundial de la Salud OPS/OMS. 2a Ed., Washington. Pp. 689695.

Aguilar M, Alvarez R. 2017. Efectividad del Triclabendazole en ovinos en la Meseta Central Santacruceña. CT INTA AER San Julián. 5 pp.
Alberghini JP. 2019. Caracterización de enfermedades del ganado para su control y prevención, en productores trashumantes de la provincia de Neuquén. Tesis de Maestría. UNCO-INTA-UNR.

Alvarez-Sánchez MA, Mainar-Jaime RC, Pérez-García J, RojoVázquez FA. 2006. Resistance of Fasciola hepatica to triclabendazole and albendazole in sheep in Spain. Vet. Rec. 159: 424-425.

Cabada MM, Lopez M, Cruz M, Delgado JR, Hill V, White AC Jr. 2016. Treatment Failure after Multiple Courses of Triclabendazole among Patients with Fascioliasis in Cusco, Peru: A Case Series. PLoS Negl. Trop. Dis. 25: 10.

Coles G, Baue C, Borgsteede FHM, Geerts S, Klei TR, Taylor MA, Waller PJ. 1992. World Association for the Advancement of Veterinary Parasitology (W.A.A.V.P) methods for the detection of anthelmintic resistance in nematodes of veterinary importance. Vet. Parasitol. 44: 35-44

Fiel CA, Steffan PE, Ferreyra DA. 2011. Diagnóstico de las parasitosis más frecuentes en rumiantes: Técnicas de diagnóstico e interpretación de resultados. Eds: Pfizer San. Anim., Programa de Control Parasitario Sustentable. 143 pp.

Hassell C., Chapman V. 2012 Case report: suspect lack of triclabendazole efficacy in liver fluke in sheep in the Taranaki. Proceedings of the Society of Sheep and Beef Cattle Veterinarians. Annual Seminar of the New Zealand Veterinary Association 4.14.1-4.14.2.

Lanari MR, Domingo E, Pérez Centeno MJ, Vázquez A, Zimerman M. 2005. Productividad del chivito criollo neuquino en el sistema tradicional. VII Congreso de la Federación Iberoamericana de Razas Criollas y Autóctonas, FIRC. Buenos Aires

Lanusse CE. 2009. Contribución fármaco-parasitológica integrada a la comprensión del fenómeno de resistencia antihelmíntica. Anales de Academia Nacional de Agronomía y Veterinaria. Pp. 354-383.

Mitchell GB, Maris L, Bonniwell MA. 1998. Triclabendazoleresistant liver fluke in Scottish sheep. Vet. Rec. 143:399.

Moll L, Gaasenbeek CP, Vellema P, Borgsteede FH. 2000. Resistance of Fasciola hepatica against triclabendazole in cattle and sheep in The netherlands. Vet. Parasitol.91: 153-8.

Mooney L, Good B, Hanrahan JP, Mulcahy G, de Waal T. 2009. The comparative efficacy of four anthelmintics against a natural acquired Fasciola hepatica infection in hill sheep flock in the west of Ireland. Vet. Parasitol. 164: 201-205.

Nari A. 2003. Resistencia de los Antiparasitarios: Estado Actual con Énfasis en América Latina. Dirección de Producción y Salud Animal, FAO, Vialle delle di Caracalla- Roma, Italia.

Olaechea FV. 2007. Fasciola hepatica. Enfermedades parasitarias de los ovinos y otros rumiantes menores en el cono sur de América. INTA EEA Anguil. Pp. 159-168.

Olaechea FV, Raffo F, Larroza M, Cabrera R. 2011. Revisión de hallazgos parasitológicos en bovinos en Patagonia realizados en el Laboratorio de Parasitología de la EEA-INTA Bariloche 2005-2010. En AAPA libro de resúmenes. Asoc. Arg. Prod. Animal. 
Oliveira DR, Ferreira DM, Stival C, Romero F, Cavagnolli F, Kloss A. Araújo F, Molento MB. 2008. Triclabendazole resistance involving Fasciola hepatica in sheep and goats during an outbreak in Almirante Tamandare, Parana, Brazil. Rev. Bras. Parasitol. Vet. 17: 149-153

Ortiz P, Scarcella S, Cerna C, Rosales C, Cabrera M, Guzmán M. 2013. Resistance of Fasciola hepatica against Triclabendazole in cattle in Cajamarca (Peru): a clinical trial and an in vivo efficacy test in sheep. Vet. Parasitol. 195: 118-21.

Overend DJ, Bowen FL. 1995. Resistance of Fasciola hepatica to triclabendazole. Aust. Vet. J. 72: 275-276.

Prieto A. 2016. Determinación de la eficacia clínica de antiparasitarios fasciolocidas en vaquillonas en la ciudad de Sarmiento, Chubut. Tesina de grado. Facultad de Ciencias Veterinarias UNCPBA.

Thomas I, Coles GC, Duffus K. 2000. Triclabendazole-resistant Fasciola hepatica in southwest Wales. Vet. Rec. 146:200. 